\title{
Erratum to: Pythagorean Triples, Additive Problems, and More
}

\section{Erratum to:}

\section{Chapter 4 in: E. Grigorieva, Methods of Solving \\ Number Theory Problems, https://doi.org/10.1007/978-3-319-90915-8_4}

In the original version of the book, the term "convergence" has to be updated as "congruence" under Sect. 4.3.2. in Chapter 4. The erratum chapter and the book have been updated with the change.

The updated online version of this chapter can be found at https://doi.org/10.1007/978-3-319-90915-8_4 